\title{
Belphégor
}

\section{Fantômas, personnage mobile et intertextuel : De la série française à l'œuvre cortazarienne}

\section{Marie-Alexandra Barataud}

\section{(2) OpenEdition}

\section{Journals}

Édition électronique

URL : http://journals.openedition.org/belphegor/87

DOI : 10.4000/belphegor.87

ISSN : 1499-7185

Éditeur

LPCM

Référence électronique

Marie-Alexandra Barataud, «Fantômas, personnage mobile et intertextuel : De la série française à l'œuvre cortazarienne», Belphégor [En ligne], 11-1 | 2013, mis en ligne le 05 avril 2013, consulté le 19 juin 2020. URL : http://journals.openedition.org/belphegor/87 ; DOI : https://doi.org/10.4000/ belphegor.87

Ce document a été généré automatiquement le 19 juin 2020.

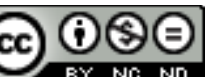

Belphégor est mis à disposition selon les termes de la Licence Creative Commons Attribution - Pas d'Utilisation Commerciale - Pas de Modification 4.0 International 


\title{
Fantômas, personnage mobile et intertextuel : De la série française à l'œuvre cortazarienne
}

\author{
Marie-Alexandra Barataud
}

1 Personnage à l'identité questionnée, homme masqué, Fantômas demeure une énigme non seulement identitaire mais également littéraire. Héros protéiforme et éclectique s'il en est un, il a inspiré de nombreuses adaptations et réécritures aussi bien dans le domaine littéraire que pictural ou cinématographique. L'agilité légendaire du héros n'a d'ailleurs d'égale que la vélocité avec laquelle les intrigues de la série française se déplacent: les récits de ses aventures n'ont cessé de voyager, d'être transmis ou adaptés hors frontières depuis le début de leur parution. Julio Cortázar s'est lui aussi pris au jeu du personnage, en offrant sa propre réinterprétation dans un texte de 1975. Fantomas contra los vampiros multinacionales ${ }^{1}$ est sans aucun doute l'une des œuvres les moins connues et les moins étudiées de l'auteur argentin, bien qu'il s'agisse d'un ouvrage hybride dont la qualité narrative et graphique sert le projet littéraire et politique de Cortázar. Riche des «modes de dérivation » chers à l'homme de littérature amateur de jeux "hypertextuels » et "hyperartistiques ", cette création embrasse un double projet : l'écriture, à la fois réécriture et adaptation, ainsi que la « utopía realizable $»^{2}$ dans laquelle se confondent et se répondent visée politique et objectif littéraire. À cette fin, Cortázar va, à partir de l'interprétation mexicaine des aventures du héros de littérature populaire française, créer un personnage qui évoluera dans une œuvremontage dont les ressemblances et les dissonances d'avec les œuvres fantômassiennes dont elle s'inspire vont permettre l'avènement d'un livre-objet original. Oscillant entre France et Mexique et s'ancrant dans la réalité latino-américaine, le Fantomas cortazarien acquiert une dimension d'actualité particulière où semblent poindre une note d'espoir et une quête qui, rejoignant les préoccupations de leur auteur, alimentent une œuvre dont le genre est ici questionné. 


\section{Mobilité géographique du récit}

2 S'il est un trait inhérent au bandit masqué, né sous la plume française, c'est son extrême mobilité. Ombre qui planait sur la ville de Paris, Fantômas est aujourd'hui un personnage dont le mythe a traversé les frontières et dont les récits parcourent le monde. Lorsque Cortázar entend parler, à Paris, de la bande dessinée mexicaine Fantomas. La inteligencia en llamas (Martré et Cruz) dont il serait l'un des protagonistes, il s'empresse de s'en procurer un exemplaire. Les aventures fantômassiennes ont traversé l'Atlantique depuis la France jusqu'au Mexique pour y être adaptées aux critères de la culture de masse nationale. Elles font ensuite le chemin inverse et sont reprises, toujours en espagnol, par un auteur argentin demeurant à Paris qui publiera sa réécriture, depuis la ville du héros, au Mexique.

3 Cette mobilité fantasmatique géographique ne se limite pourtant pas au seul héros et à la série d'ouvrages qui en découle, elle est au cœur même des thématiques développées. En effet, une fois que l'ombre du bandit commence à planer sur une nation, cette dernière se l'approprie et se familiarise avec les questionnements qui lui sont propres afin de s'adresser au plus grand nombre. Loin d'une littérature élitiste, la prose fantômassienne tire sa richesse de la diversité de ses aventures et de la multiplication de ses péripéties qui font le charme de la série sur une période aussi longue. Une fois arrivée outre-Atlantique, elle subit les modifications nécessaires à son succès : de série de romans, les aventures du héros prennent la forme de numéros de bandes dessinées publiés dans un format propre au Mexique. Et c'est l'un des exemplaires de cette bande dessinée que Cortázar utilisera pour créer son propre héros fantômassien dans une œuvre qui, loin de la série, se singularisera par son unicité et son originalité.

\section{Une fiction fruit de l'écriture, de la réécriture et de l'adaptation littéraires et historiques}

4 Fantomas contra los vampiros multinacionales. Una utopía realizable narrada por Julio Cortázar est une œuvre assez brève (77 pages) dans laquelle Julio Cortázar entreprend de créer un espace de narration dont le point de départ est la réécriture d'une bande dessinée mexicaine. Cette bande dessinée originelle fut publiée par la maison d'édition Novaro en février 1975. Il s'agit de l'un des épisodes de la série «Fantomas, La amenaza elegante » et, plus précisément, du numéro 201 intitulé «La inteligencia en llamas », élaboré par Gonzalo Martré et illustré par Victor Cruz.

5 Ayant pris connaissance de l'existence de ce fascicule où il apparaissait comme protagoniste sans qu'aucune autorisation ou demande ne lui soit parvenue, Cortázar ne met que quelques mois à réagir et à répondre à cette initiative. Il décide à son tour d'utiliser cette bande dessinée, ses héros et sa trame narrative, sans l'accord préalable des créateurs ou de la maison d'édition. Il intègre des éléments de l'épisode dans une fiction originale et propose son propre ouvrage dès juin 1975 chez Excelsior, soit quelques mois après la parution de la bande dessinée mexicaine. Celle-ci s'est offerte à lui comme le support adéquat pour explorer de nouvelles expériences littéraires, génériques, et donner à connaître, dans le même temps, un événement politique fondamental et contemporain: la divulgation des Actes de la deuxième session du Tribunal Russell ${ }^{3}$. La temporalité biographique de l'auteur qui, depuis une dizaine 
d'années, s'est réveillé à la vie politique et vient de participer à la deuxième session du Tribunal Russell à Bruxelles, correspond donc autant aux événements historiques qu'il révèle et dénonce qu'à l'instant même de l'écriture. Loin de jouer de façon hasardeuse avec le temps, l'auteur considère, comme l'écrit Ricœur que « [...] le mystère du temps n'équivaut pas à un interdit pesant sur le langage ; il suscite plutôt l'exigence de penser plus et de dire autrement» (Ricœur 489). Et c'est précisément à cette quête de l'expression libre et littéraire que nous assistons. Cortázar s'adonne depuis quelques années à l'expérimentation littéraire. Il cherche à revendiquer le droit de l'auteur de créer des œuvres originales qui dépasseraient le seul terrain de la fiction, et qui garderaient pour autant, et ce quelle que soit la thématique abordée, toute leur qualité littéraire, n'excluant en aucun cas l'humour et l'actualité historique de son œuvre. Le jeu intertextuel que Cortázar instaure à plusieurs niveaux et l'adaptation-réécriture de la bande dessinée dans le cadre de son récit de fiction semblent répondre aux exigences et perspectives nouvelles de l'auteur.

\section{Fantômas vs Fantomas : similitudes et émancipation}

La complexité hypertextuelle de ce jeu de réécriture ne s'arrête pas à une intertextualité de premier niveau. Il s'agit, pour le projet de Fantomas contra los vampiros multinacionales, de créer une fiction nouvelle à partir de la réécriture-adaptation libre des artistes mexicains de la célèbre série française de romans populaires policiers. Pierre Souvestre et Marcel Allain créent le bandit français Fantômas en 1911 et publient une série de 32 épisodes de ses aventures entre 1911 et 1913; après la mort de son compagnon, Marcel Allain continue à écrire et publie un ensemble de onze aventures au total qui paraissent tout d'abord dans des journaux et sont ensuite publiées sous forme de livres. Le personnage de Fantomas est un usurpateur d'identité, passé maître dans l'art du déguisement. Le visage de ce "génie du crime ", comme on avait coutume de l'appeler, est resté une énigme pour ceux qui le poursuivaient sans relâche: l'Inspecteur de la Sûreté de Paris, Juve, son ennemi juré, ainsi que le jeune journaliste Fandor. La jeune Hélène, fille de Fantômas et fiancée de Fandor, joue un rôle relais dans les relations entre le bandit poursuivi et ses poursuivants.

7 L'adaptation mexicaine des aventures du roman populaire français débute en mars 1966 lorsque la maison d'édition Novaro lance la série d'aventures de Fantomas (sans l'accent circonflexe des éditions françaises). Initialement, les bandes dessinées publiées par Novaro reprennent les intrigues de Souvestre et Allain: il s'agissait alors d'adaptations plus ou moins fidèles des aventures françaises traduites en espagnol, illustrées et modelées au format de bande dessinée mexicain. Le principe des vols et des poursuites policières est conservé. Seul le nom de l'inspecteur change, il s'appelle Gérard. Le personnage d'Hélène est quant à lui inexistant dans cette adaptation. Cependant, au fil du temps et avec le succès grandissant de la série (aussi bien en France qu'au Mexique d'ailleurs), le Fantomas mexicain subit des transformations et s'émancipe de plus en plus de son homophone français Fantômas : "génie du crime ", "maître de tout et de tous " comme il se qualifie lui-même. En effet, si Fantomas est toujours un voleur (avec un goût très prononcé pour l'Art), il devient philanthrope et millionnaire. Le personnage acquiert une nouvelle identité complexe, savant mélange de héros français, anglais et américains, entre le gentleman cambrioleur Arsène Lupin, le riche Batman à la morale irréprochable et Robin des Bois, voleur des riches pour 
mieux défendre les pauvres... Dans des épisodes plus tardifs, Fantomas ira jusqu'à s'opposer à la corruption des Grands de ce monde afin de protéger les opprimés. Sa générosité trouve pourtant une limite: les œuvres d'art inestimables qu'il croise et qu'il collectionne. Aussi le Fantomas mexicain apparaît-il comme un riche amalgame de Fantômas, Arsène Lupin et les super-héros américains. De fait, à l'instar de ces derniers, son refuge secret est toujours protégé et dissimulé par le brouillard qui l'entoure. Très au fait des hautes technologies, il évolue également au milieu d'outils très sophistiqués. Il va jusqu'à se doter d'un conseiller scientifique personnel, le Professeur Johannes Semo qui inventa, entre autres choses, un robot appelé C-19, assistant particulier de Fantomas ${ }^{4}$.

8 En somme, l'adaptation mexicaine devient pure création lorsque les deux auteurs mexicains cessent de retranscrire les aventures françaises de Fantômas publiées en France par Souvestre et Allain et qu'ils entreprennent d'inventer une série d'épisodes sans rapport direct avec la version originale. D'autre part, le Fantomas mexicain est accompagné de toute une équipe qui se compose essentiellement de femmes, toutes très jolies et différentes, qui portent chacune le nom d'un des douze signes du zodiaque. Enfin, soulignons que si l'inspecteur Juve français est accompagné, dans sa traque du bandit, par son acolyte journaliste Fandor, le policier mexicain Gérard n'a aucun collaborateur. Ce personnage a beaucoup plus d'importance que Juve n'en a dans la série française. Ce policier dont les méthodes sont très traditionnelles n'a pas recours aux mêmes stratagèmes que Juve qui n'hésite pas à se déguiser lui aussi pour confondre Fantômas. Il n'y a donc aucune chance dans la version mexicaine de se méprendre quant à l'identité de l'inspecteur et du bandit. L'épisode « La inteligencia en llamas » du numéro 201 de la série signera le point de départ de la fiction de Cortázar à laquelle il ajoute une dimension nouvelle annoncée dès le sous-titre : l' "utopie réalisable " ${ }^{5}$, un projet complexe tant au niveau littéraire que politique dans lequel la narration multiple tient un rôle essentiel.

\section{Fantomas mexicain et Fantomas cortazarien}

La narration à niveaux multiples, dans laquelle le lecteur est immédiatement plongé, se cristallise dans les voix de l'auteur-narrateur (voix narrative omniprésente, omnipotente et omnisciente), du narrateur-protagoniste du train ("le narrateur", Julio) et celle du protagoniste Julio Cortázar de la bande dessinée Fantomas achetée un peu plus tôt par le narrateur-protagoniste à la gare. Ces trois niveaux narratifs se mélangent, s'entrechoquent et se superposent tout au long de l'œuvre créant ainsi une temporalité plurielle.

La réécriture cortazarienne esquisse une temporalité narrative qui, bien qu'amenée à évoluer au fil du récit, suit la progression du protagoniste appelé dès la première ligne du texte « le narrateur». Ce temps du récit trouve un ancrage précis à Bruxelles où le narrateur vient d'assister à la deuxième session du Tribunal Russel. Il est sur le point de prendre le train pour Paris quand il décide d'acheter une revue ou un journal pour lire pendant le trajet. Étonnamment, il ne trouve que des revues mexicaines et finit par choisir « una revistita llena de colorinches en cuya tapa un gentleman de capa violeta y máscara blanca se lanzaba de cabeza hacia el lector como para reprocharle tan insensata compra $»^{6}$ (Fantomas 13). Ce narrateur-personnage, encore seulement protagoniste, accepte la règle du jeu imposée par la bande dessinée : « leer figurita por 
figurita sin apurarse como manda la experiencia del placer » (Fantomas 14). Il se plie au jeu de la temporalité fractionnée du texte, le temps se décompose en planches puis en vignettes... Par le truchement de la bande dessinée, le protagoniste appelé «narrateur » justifie dans un premier temps sa dénomination en ce que, narrateur-lecteur, il informe le lecteur-récepteur de ce qu'il lit ${ }^{7}$. Ce narrateur-protagoniste, encore anonyme, sert la narration, de prime abord, en introduisant la bande dessinée dans le texte dès lors qu'il accepte le pacte littéraire de celle-ci. Mais, au cours de sa lecture, lorsque les images de la bande dessinée cessent d'apparaitre dans le texte et que nous avons identifié le personnage du train comme étant Julio Cortázar lui-même, du fait, dans un premier temps, des dessins de la bande dessinée (Fantomas 29) ${ }^{8}$, puis grâce à Octavio Paz qui est, tout comme Susan Sontag ou Moravia, un protagoniste tant de la bande dessinée que du récit textuel de Cortázar, qui le nomme en disant «Julio » (Fantomas 39), le narrateur apparaît alors en tant que tel dans le hic et nunc de la narration.

Ce premier temps de la narration se termine par un constat d'échec de Fantomas ; c'est alors que Cortázar intervient en tant qu'auteur, et prend à son compte l'élaboration d'une fin de l'histoire réinventée. L'impact visuel "transartistique $»^{9}$ des collages d'images et de documents en noir et blanc ${ }^{10}$ permet au lecteur de naviguer d'une temporalité à l'autre sans qu'il y ait pour autant une rupture narrative. Cortázar réussit ainsi à créer une homogénéité texte-iconographie malgré la fluctuation des fonctions de ces intégrations dans le corps du texte. En effet, celles-ci peuvent servir à expliciter, illustrer et/ou enrichir le récit. Elles révèlent, par exemple, la représentation visuelle des transformations de Fantomas ${ }^{11}$.

12 Le lecteur se voit confronté à deux fictions qui se superposent et dont les espacestemps se démultiplient: la fiction de la bande dessinée (mexicaine) qui se termine et celle du récit-fiction des nouvelles aventures (cortazariennes) de Fantomas qui, après s'être trompé de coupable va tenter de trouver la vérité. Dans cette dernière, comme pour convaincre encore un peu plus le lecteur de la complexité de l'œuvre dorénavant double, apparaissent des éléments de la réalité biographique de l'auteur renforçant ainsi la sensation d'amalgame entre temps narratif et historique que la présence de Cortázar, Sontag, Paz, Moravia ainsi que les références contemporaines au Tribunal Russell ${ }^{12}$ avaient pu provoquer.

D'autre part, la relation qui s'établit entre les différents événements et/ou les différents moments de la narration ne respectent pas une chronologie ou un ordre préétabli. En effet, au moment de la conversation téléphonique entre Julio et Susan Sontag, se dessine un épisode proleptique à caractère fantastique. Susan parle à Julio, constate et s'étonne qu'il ignore les événements qu'elle lui relate alors même que Fantomas est censé avoir appelé ce dernier avant elle (Fantomas 25). Jusqu'alors, Julio était un lecteur qui transmettait les données de sa lecture, un lecteur passif qui retranscrivait ce qu'il voyait et lisait dans le train. Il devient subitement acteur bien qu'il reste ignorant des événements de la bande dessinée tant que le pacte de lecture n'est pas rempli (lire jusqu'au bout, vignette par vignette). Se dessinent donc deux niveaux de la fiction: celui de la bande dessinée Fantomas et celui de la réalité du narrateur-protagoniste qui se superposent, celui de la bande dessinée allant faire partie de l'histoire qui va suivre. C'est le passage entre deux réalités : de la réalité du train et de la fiction de Fantomas, nous passons à une réalité mixte qui intègre le personnage de Fantomas en son sein créant ainsi un véritable jeu narratif et temporel. A l'instar de la métalepse telle que la définit Genette ${ }^{13}$, nous assistons à une fusion des deux niveaux 
narratifs qui viennent à n'en former plus qu'un même si la vraisemblance semblait annihiler cette possibilité.

\section{Un support mobile et évolutif}

14 Ce jeu de collages et de réécritures pose la question du genre pour le Fantomas de Cortázar qui est l'adaptation littéraire d'une bande dessinée mexicaine, elle-même réécriture d'un roman français.

15 En effet, lorsqu'il décide de réécrire la bande dessinée mexicaine créant ainsi sa propre œuvre, Cortázar doit sélectionner les planches qu'il inclut ou non dans sa création. Audelà de la création à proprement parler, l'auteur s'amuse, dans un jeu qui lui est propre, à expliciter son mode de sélection au sein même de la fiction dans laquelle les planches s'insèrent. En effet, au moment de la conversation téléphonique entre Julio et Susan, l'auteur ôte une partie de la conversation et se justifie par le biais de pensées du narrateur-protagoniste Julio :

Lo malo en este tipo de diálogo, solía decirse el narrador, es que prolongan muchas páginas porque se componen sobre todo de monosílabos, gritos, preguntas espasmódicas, inicios de explicación cortados por nuevas preguntas, y tendencia recíproca a insultarse por falta de rapidez mental. Todo eso sucedió tal cual, pero podía resumirse de todas maneras en una frase de Susan [...]. (Fantomas 25) ${ }^{14}$

Quoi qu'il en soit, force nous est de constater que toutes les planches de la bande dessinée, collées dans le texte de Cortázar, sont introduites par une phrase inachevée et/ou les deux points de ponctuation: aussi les vignettes de la bande dessinée n'interviennent pas dans le texte en tant qu'illustration mais bien en tant que continuité illustrée et narrée de l'histoire et de la narration principale du début du récit. Si nous considérons la première apparition d'une planche complète de la bande dessinée dans le texte cortazarien (Fantomas 15$)^{15}$, le passage du récit textuel à la bande dessinée n'apparaît que visuellement: le temps des deux espaces ne semble pas changer, ils ne se choquent ni ne se contrarient, ils se confondent en un seul temps de la narration.

Quand arrive le moment où la réalité de la bande dessinée et celle de la fiction se superposent, les planches de la bande dessinée disparaissent de l'œuvre de Cortázar en une fusion des temps narratifs, les protagonistes servant de fil rouge à la narration. Cortázar, en réinventant la fin de l'aventure de Fantomas, opte pour une série de collages en noir et blanc (16 au total) qui illustre son récit et son projet littéraire et politique. Chaque montage opère comme une association libre entre discours littéraire et élément iconographique en décomposant l'espace de l'œuvre cortazarienne pour créer une atmosphère de collage surréaliste, collages tant picturaux que spatiotemporels qui dénoncent l'actualité latino-américaine. La brèche est alors ouverte pour faire entendre la voix universelle et anonyme latino-américaine qui, défendant l'utopie, résonne en écho lors d'une conversation téléphonique grésillante.

\section{Des voix pour l'espoir}

L'ouvrage, hybride, transgresseur de normes, transatlantique et pourtant parisien, mobile et immobile à la fois, laisse cependant planer l'espoir dans le jeu conclusif de l'enfant blond à la fin du récit cortazarien : 
Por el agujero de la ventana miró hacia la calle desierta ; sentado en el cordón de la vereda un niño rubio jugaba con una piedritas. Jugaba muy seriamente, como hay que jugar; juntaba las piedritas, las tiraba entre sus pies tratando de que se entrechocaran, volvía a juntarlas, las tiraba de nuevo.

El narrador vio que Fantomas, de pie en el tejado de la casa de enfrente, miraba también al niño. [...]

El niño siguió jugando, y el narrador vio que el sol de la mañana caía sobre su pelo rubio. (Fantomas 66-67)

À l'aurore de la voix anonyme populaire universelle ${ }^{16}$ qui interfère lors de la communication téléphonique finale entre Susan et Julio (Fantomas 59-66), semble répondre le jour lumineux qui se lève sur le jeu de l'enfant anonyme que Cortázarprotagoniste et Fantomas observent. L'enfant ne cesse de répéter son geste : pour tout enfant, comme pour Cortázar, le jeu est quelque chose de très sérieux. Fantomas et Cortázar qui le regardent peuvent voir dans l'abnégation de cet enfant une métaphore humaine de ce que pourrait être leur projet politique ${ }^{17}$, cette utopie réalisable en laquelle ils croient et pour laquelle ils agissent entre monde réel et fictif superposant leur temporalité propre. Par-delà le jeu conclusif et sentencieux, l'essence même de l'action humaine semble être d'essayer et de continuer à essayer. Le temps se répète dans la réitération des gestes et des espoirs humains ouvrant les portes d'un possible futur, encore embryonnaire, dans le présent de la fiction et la réalité de son auteur.

\section{Fantomas : un objet littéraire non identifié}

Cette œuvre, narration atypique à la temporalité multiple, peut sans conteste se voir qualifiée d'intertextuelle et de transartistique. L'auteur a créé un « objet littéraire non identifié» dont la transgénéricité est devenue sa réussite majeure. Cet hybride générique novateur ${ }^{18}$ se nourrit tant des différents niveaux narratifs que de la variété des collages, des variations artistiques, de leur intégration dans le texte et de leur rôle.

Il s'agit d'un texte avec un format de publication qui le rapproche des bandes dessinées mexicaines (format de 80 pages, $16,8 \times 23,5 \mathrm{~cm}$ avec une reliure simple de deux agrafes). Cependant, dans le texte, l'œuvre de Cortázar n'en respecte ni les normes ni la forme de la narration. Le choix de Cortázar de ce genre comme support de son travail souligne et réaffirme, malgré tout, les convictions de Susan qui a réalisé la force de la bande dessinée :

El personaje de Susan reconoce las posibilidades del cómic como medio de comunicación para legar a todo el mundo. [Entonces Julio] hace la conexión entre el malo del cómic (un tal Steiner) y los condenados por el tribunal porque los condenados son los verdaderos cerebros del mal. (Merino) ${ }^{19}$

Le support original est donc un point de départ de la réalisation d'une œuvre qu'Ana Merino qualifie d'« ensayo-cuento" (Merino) alors même qu'Annabel Audureau lui préfère le terme de "roman" (Audureau). Cependant, même si Cortázar écrit une œuvre dont le genre reste non identifié ou pour le moins difficilement identifiable, il n'abandonne pas son style propre d'écriture, son goût pour le jeu considéré avec tout le sérieux qu'il requiert ainsi que sa richesse fantastique qui sert de lien entre les moments clés de son récit. 
Les parcours géographico-littéraires de Fantômas ont fini par façonner un personnage insaisissable car changeant au gré des auteurs qui l'ont créé puis repris puis réinventé. Voyageant par-delà les océans, d'aventures romanesques en péripéties dessinées, Fantômas s'est enrichi de transformations culturelles, géographiques et littéraires. Cortázar, comme pour mieux exacerber une complexité latente, a unifié les deux supports d'expression français et mexicain pour créer une œuvre-objet rendant ainsi hommage à la mobilité du héros. Partie d'une riposte, la réécriture s'est complexifiée et intensifiée tant au niveau narratif que temporel pour s'achever, comme un clin d'œil, sur un jeu d'enfant. Ouvrage ludique à reconstruire depuis la narration jusqu'au personnage, des mots jusqu'aux images, ce livre presque puzzle appelle le lecteurrécepteur à la réflexion mais aussi, et avant tout, au jeu ; activité sérieuse qui véhicule l'abnégation. À l'instar de l'enfant, et métaphoriquement du peuple sud-américain, le lecteur-récepteur est happé par le jeu littéraire dont Cortázar a tissé la toile. Et si l'œuvre n'était qu'un éternel recommencement, un essai en somme, reviendrait alors au lecteur de jongler avec les pages, de trouver son fil d'Ariane et de s'y astreindre. Rien n'est acquis, tout est à faire ou à refaire, à produire ou à reproduire, tel un travail d'auteur.

\section{BIBLIOGRAPHIE}

AUDUREAU, Annabel, Communication : «La fonction de dénonciation de l'image dans Fantomas contra los vampiros multinacionales de Julio Cortázar ", 132e congrès, Arles, 2007 et Fantômas : un mythe moderne au croisement des arts, thèse sous la direction d'Alain-Michel Boyer, 2007.

BASSO, Lelio, «I giovani e il Tribunale Russell » in Religione e scuola, $\mathrm{n}^{\circ}$ 1, 1974, consultable sur www.fondazionebasso.it.

CORTÁZAR, Julio, Fantomas contra los vampiros multinacionales. Una utopía realizable narrada por Julio Cortázar, México : Excelsior, juin 1975.

CORTÁZAR, Julio, Fantômas contre les vampires des multinationales, traduction d'Ugné Karvelis, Paris : La Différence, Collection Les Voies du Sud, 1991 [5 ${ }^{\text {ème }}$ édition].

CORTÁZAR, Julio, Rayuela, Buenos Aires : Editorial Sudamericana, 1973 (15ª edición) [1963].

CORTÁZAR, Julio, "recortes de prensa" in Queremos tanto a Glenda, Madrid : Alfaguara, 1997, [1980].

GENETTE, Gérard, Figures III, Paris : Editions du Seuil, 1972.

GENETTE, Gérard, Palimpsestes, Saint-Amand : Éditions du Seuil, 1982.

MARTRÉ, Gonzalo y CRUZ, Victor, « La inteligencia en llamas », en Fantomas, La amenaza elegante, $n^{\circ} 201$, México : Novaro, février 1975.

MERINO, Ana, "Fantomas contra Disney", in Revista latinoamericana de estudios sobre la historieta, vol 1, n 4, diciembre de 2001, pp. 203-224, http://www.rlesh.110mb.com/04/04_merino.html. 
PAREDRO, Manolo, “Un'utopia realizzabile : l'incontro tra Fantômas e Julio Cortázar", DALL'ASTA, Monica, Fantômas : la vita plurale di un antieroe, Pozzuolo del Friuli (UD) : Il Principe Costante Edizioni, 2004.

RICCEUR, Paul, Temps et Récit, 3 - Le temps raconté, Paris : Éd du Seuil, 1985.

\section{NOTES}

1. Dorénavant les références à cette œuvre apparaîtront dans le corps de texte (Fantomas page). Soulignons ici la différence orthographique entre le Fantômas français accentué sur le «o » et le Fantomas mexicain et cortazarien non accentué (pour des raisons inhérentes à la langue: l'accent circonflexe n'existe pas en espagnol). Il ne s'agit en aucun cas d'une erreur orthographique mais bien d'une distinction volontaire de notre part dans cet article.

2. "La utopía realizable » annoncée dès le titre de l'ouvrage se résume effectivement à deux aspects de la quête de l'auteur: la quête littéraire et le questionnement politique. Elle est la volonté double et non antagonique que revendique Cortázar: être un auteur engagé politiquement et produire des œuvres littéraires de qualité dont la réalité historique et culturelle latino-américaine n'est pourtant pas nécessairement exclue. Nous n'irons pas plus avant dans l'analyse politique de cette œuvre de Cortázar car le personnage de Fantomas n'intervient pas directement dans cet aspect et d'autres travaux l'ont déjà mis en exergue.

3. À cette époque de sa vie, l'auteur argentin s'est éveillé à la vie politique et s'investit dans la réalité historique et politique de l'Amérique latine et tente de mettre en relation objectif littéraire et devoir de participation à la vie politique et sociale des pays latino-américains alors en crise. Cortázar rentrait des sessions plutôt décevantes du Tribunal Russel de 1975 lorsque lui vint l'idée d'utiliser le support de la bande dessinée pour raconter une autre histoire dans laquelle apparaîtrait l'Histoire, celle de l'oppression en Amérique latine. «El primer Tribunal Russell tuvo lugar por la iniciativa del filósofo inglés del mismo nombre y se preocupó entre los años 1966 y 1967 de los crímenes cometidos por el ejército estadounidense en Vietnam. A partir de 1971, Lelio Basso que era senador socialista independiente se dedicó a la creación de un segundo Tribunal Russell que presidía. Dedica una peculiar atención a la constitución del Tribunal permanente de Popoli que se desarrolló en Bolonia en julio de 1979 » (La traduction est nôtre) (PAREDRO 213-230). Concernant le déroulement et l'aboutissement du Tribunal Russell I, l'article de Basso est consultable sur le site internet : www.fondazionebasso.it (BASSO 68-72).

«I lavori del Tribunale Russell II si svolgono in tre sessioni distinte. La prima si tiene a Roma nella primavera del 1974; la seconda a Bruxelles nel gennaio del 1975 e la terza di nuovo a Roma nel gennaio del 1976. Oltre al Brasile e al Cile (il tribunale viene fondato a due mesi dal golpe di Pinochet), gli altri paesi interessati sono: Bolivia, Santo Domingo, Puerto Rico, Haiti, Uruguay, Paraguay, Guatemala, Colombia e Argentina. » (PAREDRO 216).

4. Remarquons ici son unique problème (et pour le moins comique): il s'avère incapable de prononcer le nom de son créateur.

5. La «utopía realizable » annoncée dans le titre n'est pas clairement explicitée dans Fantomas mais tous les traits caractéristiques de ce concept y sont présents: projet littéraire, réalité historique et positionnement politique côtoie la fiction. Cortázar défend et explicite cette utopie dans des interviews postérieures...

6. " [...] une revue pleine de couleurs criardes avec une couverture où un gentleman en cape violette, masqué de blanc, se précipite la tête la première sur le lecteur, comme pour lui reprocher un achat aussi insensé [...]», CORTÁZAR traduction de Karvelis 15). Toutes les traductions que nous proposerons seront extraites de la même édition traduite par Ugné Karvelis. 
7. Cet ouvrage qu'il lit n'est autre que la bande dessinée Fantomas. La inteligencia en llamas parue chez Novaro en 1975 et dont Cortázar colle des vignettes dans son ouvrage.

8. Nous proposons ici un lien vers le site literatura.org qui offre gratuitement en ligne une version du Fantomas de Cortázar. Cette publication ne remplace cependant pas la lecture de l'originale étant donné qu'elle est entièrement en noir et blanc et qu'elle ne respecte pas la mise en page de la publication de 1975. Elle a cependant le mérite d'exister et de nous permettre d'illustrer, même si de façon partiellement satisfaisante, notre propos. Chaque fois que nous donnerons une référence aux images présentes dans le Fantomas de Cortázar, nous proposerons le lien internet de la page correspondant à l'image à laquelle nous faisons référence. Ici : http:// www.literatura.org/Cortazar/Fantomas/f9.html.

9. Selon la terminologie de Genette (Palimpsestes, 1982).

10. Les collages d'images et de photomontages en noir et blanc apparaissent dès la page 19 de l'édition originale et alternent avec les planches et/ou vignettes de la bande dessinée mexicaine avant d'être les seules à figurer dans le texte à partir de la page 40, lorsque Cortázar décide de prendre à son compte la fin de la fiction. Nous pouvons en voir des exemples aux pages dont voici les liens internet : page 45 : http://www.literatura.org/Cortazar/Fantomas/f14.html (en réalité, dans l'édition originale, cette vision de l'œil occupe une page complète et non quelques centimètres de la page !) ; p. 48, 49 et 50 qui apparaissent condensées sur deux pages internet révélant ainsi le non respect de la mise en forme initiale : http://www.literatura.org/Cortazar/ Fantomas/f14.html http://www.literatura.org/Cortazar/Fantomas/f15.html.

11. Comme c'est le cas des pages 52 à 56. Consultez http://www.literatura.org/Cortazar/ Fantomas/f16.html et http://www.literatura.org/Cortazar/Fantomas/f17.html pour avoir un aperçu des représentations du visage de Fantomas tel qu'il est proposé dans l'ouvrage de Cortázar.

12. À cette époque de sa vie, l'auteur argentin s'est éveillé à la vie politique et s'investit dans la réalité historique et politique de l'Amérique latine et tente de mettre en relation objectif littéraire et devoir de participation à la vie politique et sociale des pays latino-américains alors en crise. Cortázar rentrait des sessions plutôt décevantes du Tribunal Russel de 1975 lorsque lui vint l'idée d'utiliser le support de la bande dessinée pour raconter une autre histoire dans laquelle apparaîtrait l'Histoire, celle de l'oppression en Amérique latine. «El primer Tribunal Russell tuvo lugar por la iniciativa del filósofo inglés del mismo nombre y se preocupó entre los años 1966 y 1967 de los crímenes cometidos por el ejército estadounidense en Vietnam. A partir de 1971, Lelio Basso que era senador socialista independiente se dedicó a la creación de un segundo Tribunal Russell que presidía. Dedica una peculiar atención a la constitución del Tribunal permanente de Popoli que se desarrolló en Bolonia en julio de 1979 » (La traduction est nôtre) (PAREDRO 213-230). Concernant le déroulement et l'aboutissement du Tribunal Russell I, l'article de Basso est consultable sur le site internet : www.fondazionebasso.it (BASSO 68-72).

«I lavori del Tribunale Russell II si svolgono in tre sessioni distinte. La prima si tiene a Roma nella primavera del 1974; la seconda a Bruxelles nel gennaio del 1975 e la terza di nuovo a Roma nel gennaio del 1976. Oltre al Brasile e al Cile (il tribunale viene fondato a due mesi dal golpe di Pinochet), gli altri paesi interessati sono: Bolivia, Santo Domingo, Puerto Rico, Haiti, Uruguay, Paraguay, Guatemala, Colombia e Argentina. » (PAREDRO 216).

13. «Tous ces jeux manifestent par l'intensité de leurs effets l'importance de la limite qu'ils [les auteurs] s'ingénient à franchir au mépris de la vraisemblance, et qui est précisément la narration (ou la représentation) elle-même; frontière mouvante mais sacrée entre deux mondes : celui où l'on raconte, celui que l'on raconte. » (GENETTE, Figures III 245).

14. "L'ennui avec ce genre de dialogues, se dit le narrateur, c'est qu'ils s'étalent sur des pages et des pages, vu qu'ils se composent essentiellement de monosyllabes, de cris, de questions spasmodiques, de débuts d'explications coupées par de nouvelles questions, avec une tendance réciproque à insulter l'autre en lui reprochant son manque de rapidité mentale. Tout ceci s'est 
bien passé comme ça, mais peut être résumé en une phrase de Susan [...]», (CORTÁzAR traduction de Karvelis 27).

15. http://www.literatura.org/Cortazar/Fantomas/f3.html. L'original est en couleurs mais occupe effectivement une page complète.

16. Et c'est évidemment là la différence majeure entre la bande dessinée mexicaine et l'ouvrage cortazarien: l'ancrage dans la réalité historique et politique de l'Amérique latine. La voix anonyme populaire du peuple latino-américain crée, en effet, des interférences dans la conversation téléphonique des protagonistes cortazariens et se font l'écho des préoccupations politiques et sociales du peuple en attente du verdict du Tribunal.

17. Un lecteur du Fantômas français, dans sa variante terrifiante ou comique (l'avatar d'Hunnebelle), ne peut deviner la recharge poético-utopique de cette figure du crime sur le plan politique dans la version cortazarienne. C'est au fil de sa lecture qu'il perçoit grâce aux images (les photomontages en noir et blanc, les copies de documents ou la valeur symbolique de l'arme...) et à la portée critique de certains éléments rapportés (les Actes du Tribunal Russell II de Bruxelles, la voix latino-américaine...) la charge politique et dénonciatrice de cette récriture. Le lecteur qui connaît l'œuvre de Cortázar et son parcours personnel ne saurait, pour sa part, être surpris de la dimension critique et engagé de ce texte qui s'inscrit dans la lignée de la quête littéraire de l'auteur à l'époque. Il revendique, en effet, le droit d'écrire des œuvres littéraires dont le contenu, révélateur d'un engagement fort, ne trahirait pas la qualité. Il expérimente, depuis quelques années déjà, le recours à des médias autres que le texte pour créer des œuvres hybrides dans lesquels message verbal et visuel établissent des ponts. Dans Fantomas, Cortázar poursuit ses expériences transartistiques et semble, dans le même temps s'attaquer aux abus de pouvoir, à l'enrichissement des états les plus riches sur le compte des plus pauvres qu'ils exploitent. Il dénonce les violences et le nombre de victimes. Son propos, loin de se limiter à une revendication politique, tend à révéler une requête littéraire dont la dimension humaine ne saurait échapper au lecteur. Il aspire, en effet, au réveil des intellectuels latino-américains qui doivent, selon lui, se confronter à la réalité sociale et politique de leurs pays.

18. Genre hybride novateur mais, cependant, pas nouveau dans l'œuvre de Cortázar qui a commencé ses expérimentations littéraires dans ce domaine dans des œuvres antérieures tel que, par exemple, son roman Rayuela et qu'il continuera d'employer dans des œuvres postérieures comme c'est le cas dans la nouvelle « recortes de prensa ».

19. La traduction est nôtre: "Le personnage de Susan reconnaît les possibilités de la bande dessinée comme moyen de communication pour toucher tout le monde. [Alors Julio] fait la connexion entre le méchant de la bande dessinée (un certain Steiner) et les personnes condamnées par le Tribunal parce que les condamnés sont les véritables cerveaux du mal ».

\section{RÉSUMÉS}

A faire

INDEX

Mots-clés : Fantômas, Cortazar Julio, comics, politique, légitimité 
AUTEUR

MARIE-ALEXANDRA BARATAUD

Université de Limoges - EHIC 